understand known correlations between coronal X-ray fluxes, chromospheric line fluxes and stellar rotation rates. These must be controlled ultimately by the nonthermal heating and the energy loss processes. A wide range of more exotic stellar objects, including symbiotic stars, AM Herculis binary stars, and cataclysmic variables, should also provide exciting new data (A.K. Dupree, S.J. Kenyon and J.C. Raymond, Harvard-Smithsonian Center for Astrophysics; D.Q. Lamb, University of Chicago).

$P$. Gondhalekar is at the Rutherford Appleton Laboratory, Didcot OX11 OQX, and C. Jordan is in the Department of Theoretical Physics, University of Oxford, Oxford OX1 3NP, UK. UBIQUITIN

\title{
A marriage of convenience or necessity?
}

\section{Jonathan R. Warner}

Ubiourrin, a 76-amino-acid protein, is, as its name implies, ubiquitous in eukaryotic cells (see ref. 1 for a review). A complex enzymatic system attaches it to proteins, usually by peptide bond formation with the $\varepsilon$-amino of a lysine, either singly or in branched multiple ubiquitin structures. Ubiquitin can be a signal for the proteindegradation system of cells, much as a blaze on a tree serves as a signal for the logger. But in others its embrace is less lethal; it is reversibly attached to the histone $\mathrm{H} 2 \mathrm{~A}$ without causing its degradation ${ }^{2}$. Its function here is unknown.

Ubiquitin is remarkably conserved through evolution, with a similarity of 96 per cent between yeast and human', and between yeast and Trypanosoma cruzií. Even more remarkable is the conservation in the arrangement of the genes encoding ubiquitin, which are found in two basic forms in most organisms (see figure). Class I is a polyubiquitin gene which encodes a polyprotein of up to 100 uninterrupted, tandemly repeated ubiquitins presumably released by proteolysis at the Gly-Met peptide bond that joins the repeats. Class II is a fusion between a single ubiquitin and one of two other sequences, of either 52 or $76-80$ predominantly basic amino acids, again remarkably conserved through evolution. In this issue these basic sequences are identified, in the yeast Saccharomyces cerevisiae by Finley et al. "on page 394, and in mammals by Redman and Rechsteiner' on page 438 , as members of another common group, ribosomal proteins. Finley et al. "fused antigenic tags to the $U B I I$ and the $U B I 3$ gene products of yeast and find the 52amino-acid $U B I I$ tail in the $60 \mathrm{~S}$ ribosomal subunits and the 76-amino-acid UBI3 tail in the $40 \mathrm{~S}$ subunits. A protein with the amino-terminal sequence of the UBI3 extension had previously been identified as ribosomal protein S37 (ref. 8). By immunoblotting with antibodies made against a peptide within the mammalian 8()-amino-acid extension, Redman et al. find the same protein in the $40 \mathrm{~S}$ subunits and identify it as mammalian ribosomal protein S27a. Although less conserved through evolution than ubiquitin, yeast S37 and mammalian S27a are similar. Protein sequence data indicate that the ribosomal proteins from both organisms have been cleaved from the ubiquitin moiety without the loss of any amino acids.

By deletion analysis, Finley et al." show that the 52-amino-acid ribosomal protein is essential for cell growth, as are all other Translation product Final product Yeast gene

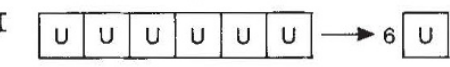
UBI4 II u
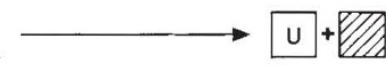

UBI3

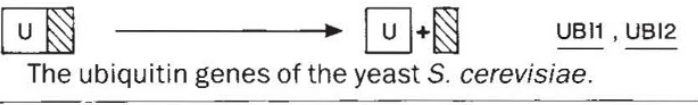

eukaryotic ribosomal proteins they tested. It is therefore surprising that cells without the gene encoding ribosomal protein S37 do grow, although poorly. These cells have a severe deficiency of 40 S ribosomal subunits, apparently because of a defect in the final processing step(s) in the formation of $18 \mathrm{~S}$ ribosomal RNA. Does the lack of S37 influence only ribosome assembly, or does it also have some effect on the rate or accuracy of translation?

It is a remarkable finding that the sole source of two ribosomal proteins is derived from ubiquitin fusion genes. But it is truly extraordinary that this arrangement has persisted throughout eukaryotic evolution, even into the freakish trypanosomes. Is ubiquitin dependent on ribosomal proteins or vice versa? Or is there a mutual symbiosis?

Introns are rare in the genes of $S$. cerevisiae, whereas most of the genes encoding ribosomal proteins have a single intron. Although it is not yet clear what selective pressure has maintained introns in the ribosomal protein genes, it is interesting that the members of the duplicate pair, $U B I 1$ and $U B I 2$, each have an intron in the ubiquitin-coding portion of the gene.

Another conserved region of ubiquitin genes is the heat-shock promoter element ${ }^{3}$. In several organisms, including yeast, the polyubiquitin genes are responsive to stress, but the fusion genes are constitutively expressed. In the case of the yeast genes, this is presumably because of the ribosomal protein enhancer elements, UAS ${ }_{\text {rpu, }}$ found upstream of the fusion genes ${ }^{2}$ and almost all ribosomalprotein genes the main source of ubiquitin during $\log$ growth, when ribosome synthesis is active. The polyubiquitin gene, UBI4, is derepressed only late in the growth cycle. during nitrogen starvation or after heat shock - all conditions under which ribosomal protein synthesis is repressed. Ubiquitin need not be derived from the fusion genes, however. If ubiquitin sequences are deleted from all the fusion genes, the cell still grows simply by turning on the polyubiquitin gene".

The ubiquitin moiety of $U B I 3$ is helpful but not essential in the function of ribosomal protein S37. If S37 is expressed from a $U B I 3$ gene from which the ubiquitin-coding sequences have been deleted, many copies of the mutant gene are needed to replace fully one copy of the $U B I 3$ gene. One could speculate that the ubiquitin is used as a translational spacer for a very short protein, as a nuclear localization signal or as a 'wedge' to assist in assembling proteins into the ribosome. At what point in the assembly process is the ubiquitin cleaved? An alternative view derives from the observation that the ribosomal proteins turn over very rapidly if not assembled into a ribosome ${ }^{11.12}$. Ubiquitin could protect the ribosomal proteins from degradation, or perhaps cleavage from a ubiquitin molecule is a stratagem to make a protein without an amino-terminal methionine.

Although neither ubiquitin nor ribosomal protein seems essential for the other, there must be some explanation for this ancient and enduring marriage. That explanation may reveal the original role of ubiquitin, or some hitherto unsuspected connection between protein synthesis and protein degradation.

Jonathan R. Warner is in the Department of Cell Biology, Albert Einstein College of Medicine, Bronx, New York 10461, USA.

\footnotetext{
1. Rechsteiner, M. A. Rev. Cell Biol. 3. 1-30 (1987)

2. Wu, R.S., Kohn, R.W. \& Bonner, W.M. J. biol. Chem 256 5916-5920 (1980)

Ozkaynak, E. et al. EMBO J. 6. 1429-1439 (1987)

Lund. P.K. et al. J. biol. Chem 260. 7609-7613 (1985)

5. Swindle, J. et al. EMBO J. 7. 1121-1127 (1988)

6. Finley, D., Bartel, B. \& Varshavsky, A. Nature 338. 394 401 (1989)

. Redman, K.L. \& Rechsteiner, M. Nature 338, 438-440 (1989)

8. Otaka, E., Higo, K. \& Itoh. T. Molec. gen. Genet. 195 544-546 (1984)

9. Rotenberg, M.O. \& Woolford, J.L. Molec. cell. Biol, 6 $674-687$ (1986)

10. Woudt, L.P. et al. EMBO J. 5. 1037-1040 (1986). 11. Warner J.R. J molec Biol 115, 315-333 (1977) 12. Maicas, E.F. et al. Molec cell. Biol. 8. 169-175 (1988)
} 